\title{
Prevalence, hematological findings and genetic diversity of Bartonella spp. in domestic cats from Valdivia, Southern Chile
}

\author{
ANANDA MÜLLER ${ }^{1}$ *, ROMINA WALKER ${ }^{1}$, PEDRO BITTENCOURT ${ }^{1}$, ROSANGELA \\ ZACARIAS MACHADO ${ }^{2}$, JYAN LUCAS BENEVENUTE ${ }^{2}$, RENAN BRESSIANI DO \\ AMARAL ${ }^{2}$, LUIZ RICARDO GONÇALVES ${ }^{2}$ and MARCOS ROGÉRIO ANDRÉ ${ }^{2}$ \\ ${ }^{1}$ Facultad de Ciencias Veterinarias, Instituto de Ciencias Clínicas Veterinarias, Universidad Austral de Chile, Valdivia, \\ Chile \\ ${ }^{2}$ Departamento de Patologia Veterinária, Faculdade de Ciências Agrárias e Veterinárias, Universidade Estadual Paulista \\ (FCAV/UNESP), Faboticabal, SP, Brazil
}

(Received 30 October 2016; revised 12 November 2016; accepted 14 November 2016; first published online 12 December 2016)

SUMMAR Y

The present study determined the prevalence, hematological findings and genetic diversity of Bartonella spp. in domestic cats from Valdivia, Southern Chile. A complete blood count and nuoG gene real-time quantitative PCR (qPCR) for Bartonella spp. were performed in 370 blood samples from cats in Valdivia, Southern Chile. nuoG qPCR-positive samples were submitted to conventional PCR for the $g l t A$ gene and sequencing for species differentiation and phylogenetic analysis. Alignment of $g l t A$ gene was used to calculate the nucleotide diversity, polymorphic level, number of variable sites and average number of nucleotide differences. Bartonella DNA prevalence in cats was $18 \cdot 1 \%(67 / 370)$. Twenty-nine samples were sequenced with $62 \cdot 0 \%(18 / 29)$ identified as Bartonella henselae, $34 \cdot 4 \%(10 / 29)$ as Bartonella clarridgeiae, and $3 \cdot 4 \%(1 / 29)$ as Bartonella koehlerae. Bartonella-positive cats had low DNA bacterial loads and their hematological parameters varied minimally. Each Bartonella species from Chile clustered together and with other Bartonella spp. described in cats worldwide. Bartonella henselae and B. clarridgeiae showed a low number of variable sites, haplotypes and nucleotide diversity. Bartonella clarridgeiae and B. koehlerae are reported for the first time in cats from Chile and South America, respectively.

Key words: Bartonella henselae, Bartonella clarridgeiae, Bartonella koehlerae, cat scratch disease, qPCR, South America.

\section{INTRODUCTION}

The Bartonella genus includes fastidious haemotropic Gram-negative bacteria mainly transmitted by arthropod-vectors (Chomel et al. 2009). Over the last 20 years, the number of Bartonella species or subspecies identified from a wide range of mammals has increased considerably (Chomel et al. 2009). Among the species or subspecies known or suspected to be pathogenic for humans, three have the domestic cat as their natural reservoir, namely Bartonella henselae, Bartonella clarridgeiae (Boulouis et al. 2005) and Bartonella koehlerae (MogollonPasapera et al. 2009). Bartonella henselae and $B$. clarridgeiae are associated with cat-scratch disease (CSD) and other syndromes in humans and are the most commonly identified Bartonella species in cats, worldwide (Boulouis et al. 2005; Breitschwerdt et al. 2010b). Bartonella koehlerae was previously reported as a cause of human endocarditis (Avidor et al. 2004; Chomel et al. 2009).

\footnotetext{
* Corresponding author: A. Müller, Facultad de Ciencias Veterinarias, Instituto de Ciencias Clínicas Veterinarias, Universidad Austral de Chile, Veterinary Hospital UACh, PO Box 567, Valdivia, Chile. E-mail: ananda. muller@uach.cl
}

Considering that bacteria from the Bartonella genus are fastidious to grow in vitro, serological [Indirect Fluorescent Antibody Test (IFAT) and enzyme-linked immunosorbent assay (ELISA)] and molecular [conventional and real-time quantitative polymerase chain reaction (cPCR and $\mathrm{qPCR}$ ), respectively] techniques are widely used for the diagnosis of Bartonella infection (MogollonPasapera et al. 2009). Serological tests have limited specificity due to cross-reactions and inconsistent results (Maggi et al. 2011). Molecular techniques are sensitive and allow species identification (Fenollar and Raoult, 2004). cPCR assays have limited sensitivity compared with qPCR (André et al. 2016). A pre-enrichment liquid culture medium ('Bartonella Alpha Proteobacteria Growth Medium', BAPGM) prior to PCR was suggested to improve the sensitivity of molecular techniques, mainly for detecting Bartonella species in biological samples from non-reservoir hosts, including humans (Maggi et al. 2005, 2011; Breitschwerdt et al. 2010a; Pérez et al. 2011).

The prevalence rates of Bartonella spp. detected by PCR vary considerably among cat populations, with an increase from cold ( $0 \%$ in Norway) (Bergh 
et al. 2002) to warm and humid climates (61\% in the Philippines) (Chomel et al. 1999). Although Bartonella spp. infection in cats can vary between areas, it is often associated with flea infestation (Boulouis et al. 2005). Bartonella spp. infection is associated with extended, often subclinical (Kordick et al. 1999), asymptomatic, with longlasting intraerythrocytic bacteremia in domestic cats (Chomel et al. 2009). Understanding the potential associations between Bartonella spp. infection and clinical disease in cats is complicated. There is little information about haematological abnormalities in naturally infected cats (Breitschwerdt, 2008).

In Chile, few reports have assessed the prevalence of Bartonella in cats. An overall B. henselae seropositivity of $85 \%$ was found in cats sampled in three cities (Coquimbo, Santiago and Valdivia) (Ferrés et al. 2005). Another study in Valdivia, Southern Chile, described a $B$. henselae serosurvey of $71 \%$ (Zaror et al. 2002). Additionally, 41.7\% (25/60) of blood samples from cats in Santiago, Central Chile, were culture positive for Bartonella, and confirmed as $B$. henselae by $16 \mathrm{~S}$ RNA gene sequencing (Ferrés et al. 2005). Nevertheless, the molecular prevalence of Bartonella spp. in cats from Southern Chile and its strain diversity are not yet known. Bartonella DNA was detected in $10 \cdot 8 \% \quad(4 / 37)$ of Ctenocephalides felis fleas collected from cats sampled in Chilean animal pounds (Pérez-Martínez et al. 2009). After PCR amplification and sequencing of $r p o B$, glt $A$ genes and the 16-23S rRNA intergenic transcribed spacer, the species involved were identified as $B$. clarridgeiae and $B$. henselae (PérezMartínez et al. 2009). Increased exposure to cats, particularly kittens and cat-related trauma were associated with a higher prevalence of Bartonellaassociated disease (Boulouis et al. 2005; Breitschwerdt et al. 2010a). In Chile, the disease is not of mandatory reporting. Nevertheless, more than 200 human cases of bartonellosis were diagnosed between 1997 and 2000 (Ferrés et al. 2005). According to previous studies, cats play a major role as $B$. henselae reservoirs in Chile; consequently, humans who have contact with those animals are at risk (Ferrés et al. 2005). The present study aimed at determining the prevalence, haematological findings and genetic diversity of Bartonella spp. in domestic cats from Valdivia, Southern Chile.

\section{MATERIALS AND METHOD}

\section{Animals and area of study}

The study was approved by the Universidad Austral de Chile (UACh) bioethics committee under the protocol number UACh 142/2013.

To accurately determine Bartonella spp. prevalence in Valdivia (39 $48 \quad 30 \mathrm{~S}, \quad 73 \quad 14 \quad 30 \mathrm{~W}$ ), Southern Chile, the required sample size was estimated considering a prevalence of $50 \%$, which fits the criteria when prevalence is unknown (Thrusfield, 2007), and corrected according to the cat population of Valdivia (Zuñiga, 2007), providing a sample of 370 cats. A 5\% precision and 95\% confidence interval were used (Thrusfield, 2007). Over a 15-month period (August 2013-November 2014), 370 client-owned cats had their blood sampled by a veterinary team. The cats came from all Valdivia city locations in order to acquire balanced and representative sampling. Samples were taken from: (1) cats during home visits to pet-owning households; and (2) cats admitted to the Veterinary Hospital of UACh, Valdivia (Fig. 1). Cats were sampled regardless of age, gender, health and reproductive status. Each owner signed a consent form before samples were taken.

\section{Haematological analysis}

Blood samples were collected aseptically by cephalic or jugular venipuncture, divided into two EDTA collecting plastic tubes (Vacutainer ${ }^{\circledR}$ ), and sent to the UACh Veterinary Clinical Pathology Laboratory. One EDTA anticoagulated blood sample was stored at $-20^{\circ} \mathrm{C}$ until DNA extraction/purification. The other EDTA anticoagulated blood was used to perform a complete blood count (CBC). The following parameters were analysed: red blood cell, white blood cell (WBC) and platelet counts; haemoglobin concentration; packed red cell volume; mean corpuscular volume (MCV); and mean corpuscular haemoglobin concentration. An automated haematology analyser, KX-21N (Sysmex ${ }^{\circledR}$, Japan), was used. The blood smears were stained with rapid staining (Hemacolor ${ }^{\circledR}$, Merck) for a differential WBC count.

\section{DNA extraction/purification}

Frozen EDTA blood samples were thawed at room temperature and vortexed. DNA extraction from $100 \mu \mathrm{L}$ of blood was performed using a DNeasy ${ }^{\circledR}$ Blood \& Tissue Kit $\left(\mathrm{QIAGEN}^{\circledR}\right.$, Valencia, CA, USA) and was eluted with $100 \mu \mathrm{L}$ of elution buffer, according to the manufacturer's instructions. Concentration and purity were determined using a NanoDrop ND-1000 spectrophotometer (Thermo Scientific $^{(}$, USA). The absorbance ratio 260 and $280 \mathrm{~nm}\left(\mathrm{OD}_{260} / \mathrm{OD}_{280}\right)$ provided an estimate of sample purity, accepting a ratio of $1 \cdot 8 \pm 0 \cdot 2$ as 'pure'.

\section{Endogenous control real-time PCR}

The 28S rDNA gene was used as an internal control for a PCR assay for feline genomic DNA (Helps et al. 2005) using primers feline-28S rDNAFw (5'-AGCAGGAGGTGTTGGAAGA G-3') and feline-28S rDNARv (5'-AGG GAGA GCCTAAATCAAAGG-3') to discard the presence 

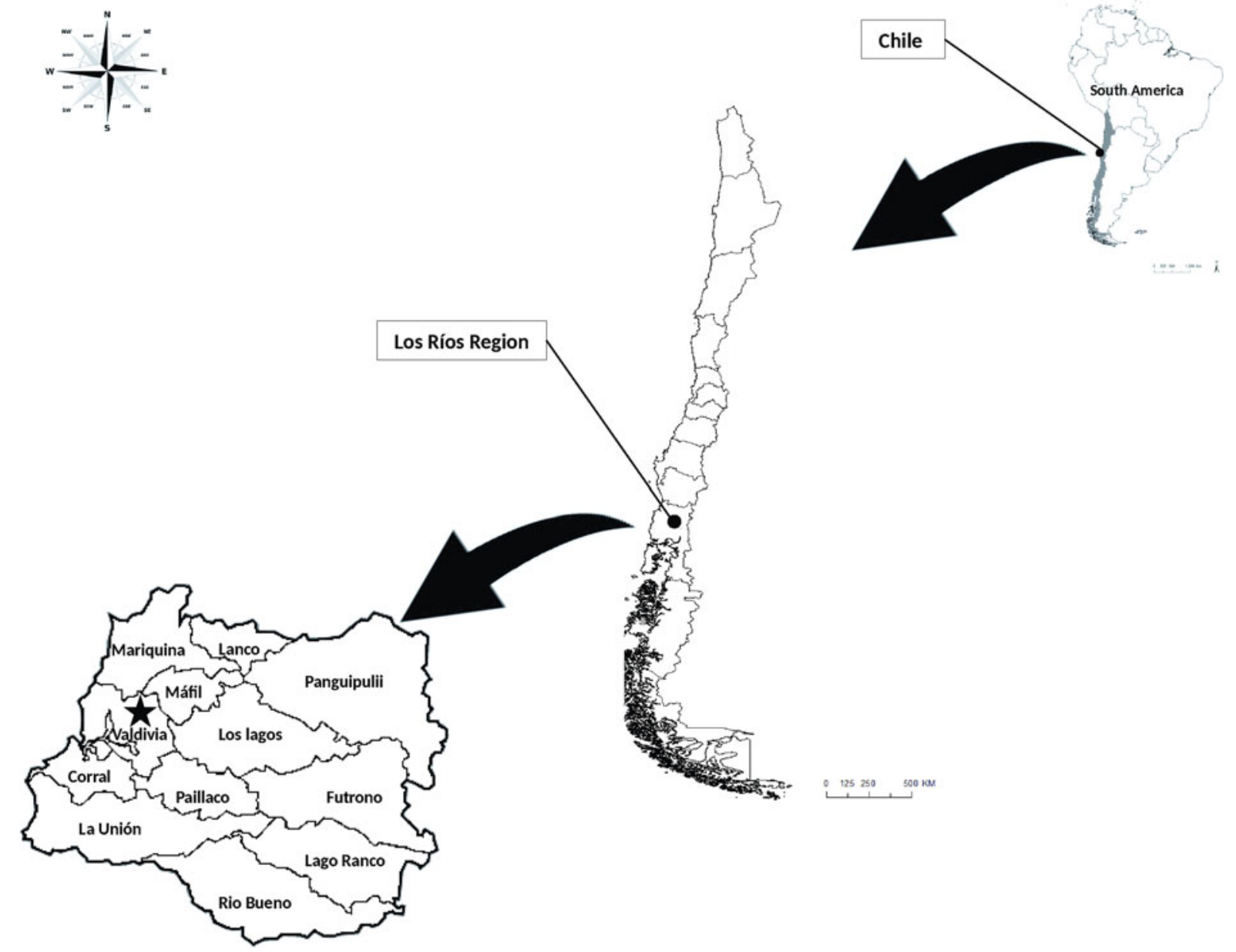

Fig. 1. Map of Chile, showing Valdivia City located in the Los Ríos Region, where samples from cats were taken (MapInfo Professional 7·5 SCP).

of PCR inhibitors. The reaction mixture was composed of $12 \cdot 5 \mu \mathrm{L}$ of Maxima ${ }^{\circledR} \mathrm{SYBR}$ Green/Rox Master Mix ('Thermo Scientific ${ }^{\odot}$, USA), $300 \mathrm{nM}$ of the forward and reverse primers and $5 \mu \mathrm{L}$ of DNA template, brought to a total volume of $25 \mu \mathrm{L}$ with nuclease-free water (Thermo Scientific $^{\odot}$, USA). The amplification conditions were $50^{\circ} \mathrm{C}$ for $2 \mathrm{~min}$ and $95^{\circ} \mathrm{C}$ for $10 \mathrm{~min}$, followed by 40 cycles of $95^{\circ} \mathrm{C}$ for $15 \mathrm{~s}$ and $60^{\circ} \mathrm{C}$ for $1 \mathrm{~min}$. Reactions were performed in a Stratagene Mx3000PTM (Agilent Technologies).

\section{$q P C R$ for Bartonella $s p p$.}

28S rDNA cPCR-positive samples were subsequently submitted to a previously described qPCR for Bartonella spp. targeting nuoG gene (André et al. 2016). Amplification reactions were performed in duplicate using $10 \mu \mathrm{L}$ of PCR mixtures containing $5 \mu \mathrm{L}$ of $\mathrm{Go} \mathrm{Taq}^{\circledR}$ Probe qPCR Master Mix, dTTP (Promega, Madison, WI, USA), 1,2 $\mu \mathrm{M}$ of each primer [F-Bart (5'-CAATCTTC'TTTTG C'TTCACC-3') and R-Bart (5'- TCAGGGCTT'T ATGTGAATAC-3'), hydrolysis probe [TexasRed-
5'-TTYGTCATTTGAACACG-3'(BHQ2a-Q)3'] and $1 \mu \mathrm{L}$ of the DNA sample. PCR amplifications were conducted in Low-Profile Multiplate ${ }^{\mathrm{TM}}$ Unskirted PCR Plates $\left(\right.$ BioRad $^{\odot}$, Hercules, CA, USA) using a CFX96 Thermal Cycler $\left(\mathrm{BioRad}^{\circledR}\right)$. The amplification conditions were $95^{\circ} \mathrm{C}$ for $3 \mathrm{~min}$ followed by 40 cycles of $95^{\circ} \mathrm{C}$ for $10 \mathrm{~min}$ and $52.8{ }^{\circ} \mathrm{C}$ for $30 \mathrm{~s}$. The qPCR was performed following the MIQE (Minimum Information for Publication of Quantitative Real-Time PCR Experiments) (Bustin et al. 2009). Amplification efficiency $(E)$ was calculated from the slope of the standard curve in each run using the following formula $\left(E=10^{-1 / \text { slope }}\right)$. Copy numbers were estimated using 10-fold serial dilutions of pIDTSMART plasmids (Integrated DNA Technologies, Coralville, IA, USA) encoding the nuoG $B$. henselae sequence (insert containing $83 \mathrm{bp}$ ). The number of plasmid copies was determined according to the formula $\left[\mathrm{Xg} \mu \mathrm{L}^{-1} \mathrm{DNA} /\right.$ (plasmid length in $\mathrm{bp} \times 660)] \times 6 \cdot 022 \times 10^{23} \times$ plasmid copies $\mu \mathrm{L}^{-1}$. Bartonella henselae DNA obtained from a naturally infected cat (Miceli et al. 2013) was used as a positive control. All PCR runs were performed with 
nuclease-free water (Thermo Scientific ${ }^{\odot}$, USA) as a negative control. Replicates showing a $C q$ difference higher than 0.5 were retested.

\section{cPCR for Bartonella $s p p$.}

For further molecular characterization and species differentiation, nuoG Bartonella qPCR-positive samples were tested using a previously described (Billeter et al. 2011) cPCR targeting a 767-bp fragment of the citrate synthase gene ( glt $A)$, using primers CS443f (5'- GCTATGTCTGCATTC TATCA-3') and CS1210r (5'-GATCYT CAATC ATTTCTT'TCCA-3'). Each DNA sample $(5 \mu \mathrm{L})$ was used as a template in $25 \mu \mathrm{L}$ reaction mixtures containing 10× PCR buffer, $1.5 \mathrm{mM} \mathrm{MgCl}_{2}$, $0.2 \mathrm{~mm}$ deoxynucleotide triphosphate (dNTPs) mixture, 0.625 U Platinum Taq DNA Polymerase (Invitrogen $^{\odot}$, Carlsbad, CA, USA), and $0.5 \mu \mathrm{M}$ of each primer. $\mathrm{cPCR}$ amplification reactions were performed using a T100 BioRad termocycler $\left(\mathrm{BioRad}^{\odot}\right)$ with the following cycling conditions: $94^{\circ} \mathrm{C}$ for 2 min; 45 cycles of $94^{\circ} \mathrm{C}$ for $30 \mathrm{~s}, 48^{\circ} \mathrm{C}$ for $1 \mathrm{~min}$ and $72{ }^{\circ} \mathrm{C}$ for $1 \mathrm{~min}$; and one cycle of $72{ }^{\circ} \mathrm{C}$ for 5 min. PCR products were separated by electrophoresis on a $1 \%$ agarose gel stained with ethidium bromide. To prevent PCR contamination, DNA extraction, reaction setup, PCR amplification and electrophoresis were performed in separate rooms. Gels were visualized under ultraviolet light using the Image Lab Software version 4.1 (BioRad $\left.^{\odot}\right)$. The reaction products were purified using the Silica Bead DNA gel extraction kit (Fermentas ${ }^{\circledR}$, São Paulo, SP, Brazil).

\section{Sequencing and Phylogenetic analysis}

Only glt $A$-cPCR-positive samples presenting strong band intensity were submitted for sequencing. Sanger sequencing was performed on purified amplified DNA fragments from positive samples in an automatic sequencer (ABI Prism 310 genetic analyser; Applied Biosystems ${ }^{\odot} /$ Perkin-Elmer) for species identification and subsequent phylogenetic analysis. Consensus sequences were obtained through analysis of the sequenced products, from both the forward and the reverse oligonucleotides, using the CAP3 program (http://mobyle.pasteur.fr/ cgi-bin/MobylePortal/portal.py). Primer sequences were trimmed from the consensus sequences prior to Blastn analysis. Comparisons with sequences in GenBank were performed using the basic local alignment search tool (BLASTn). The sequences were aligned with sequences published in GenBank using Clustal/W and manually adjusted in Bioedit v. 7.0.5.3 (Carlsbad). Phylogenetic inference based on maximum-likelihood criterion (ML) was inferred with RAxML-HPC BlackBox 7.6.3 (Statamakis et al. 2008) through the CIPRES Science Gateway
(Miller et al. 2010) estimating the proportion of invariable sites by an evolutive model GAMMA GTR + I.

\section{Nucleotide diversity}

The alignment sequences of the glt $A$ gene, amplified in the present study, were used to calculate the nucleotide diversity $(\pi)$, polymorphic level [haplotype diversity $(H d)$ ], number of variable sites $(v s)$ and the average number of nucleotide differences $(K)$ using the DnaSP v5.10 (Librado and Rozas, 2009).

\section{Statistical analysis}

To determine Bartonella spp. prevalence, qPCRpositive cats were divided by the total number of cats sampled and multiplied by 100 . The observed prevalence rates were expressed in percentages and the $95 \%$ IC was calculated. Descriptive statistics were obtained for haematological parameters and the cats were divided into two groups according to their Bartonella spp. status based on the qPCR results: Bartonella spp. negative or Bartonella spp. positive. The normal distribution of data was evaluated by a Shapiro-Wilk's test. The non-normally distributed data were analysed using the KruskalWallis test to determine if there were any significant differences between the haematological variables of the Bartonella spp. status groups. A $P$-value $\leqslant 0 \cdot 05$ was considered statistically significant. Data were analysed using RStudio version 0.99 .903 and were available for all 370 cats, except for platelet counts, which were available only for 171 cats.

\section{RESULTS}

\section{Bartonella spp. $q P C R$ results}

All 370 DNA samples [median and standard deviation (S.D.) of DNA concentration $=26 \cdot 5 \pm 12 \cdot 3 \mathrm{ng}$ $\mu \mathrm{L}^{-1}$; mean and s.D. $260 / 280$ ratio $\left.=1.79 \pm 0 \cdot 07\right]$ were positive for the feline $28 \mathrm{~S}$ rDNA endogenous gene. Molecular prevalence of Bartonella DNA in cats by qPCR (mean and S.D. efficiency of reactions: $\left.96 \cdot 1 \pm 0 \cdot 83 \%, r^{2}=0.998 \pm 0 \cdot 00046\right)$ was $18 \cdot 1 \%(67 /$ 370) $(95 \%$ CI $14 \cdot 422 \cdot 5 \%)$. Thirty-eight samples had a consistent $C q$ (mean and s.D. 30.21 $\pm 2 \cdot 93$ ) and quantification (mean $1.32 \times 10^{3}$; minimunmaximun $2 \cdot 13 \times 10^{0}-3 \cdot 19 \times 10^{4}$ nuo $G$-copies $\mu \mathrm{L}^{-1}$ ) (Table 1). Twenty-nine cats had inconsistent Bartonella-qPCR quantification assays, due to that, their $C q$ and quantification results were not registered in the present work.

\section{cPCR results and phylogenetic analysis}

Of 67 nuoG-qPCR-positive samples, 49 (73\%) were glt $A$-cPCR-positive and 29 were sequenced. 
Table 1. Bartonella spp.-positive cat blood samples with their respective $C q$ (cycle of quantification) and quantification (nuoG-copies $\mu \mathrm{L}^{-1}$ ) mean values obtained by qPCR assays, and the Bartonella species identified by BLASTn analysis

\begin{tabular}{|c|c|c|c|c|c|c|}
\hline \multirow[b]{2}{*}{$\begin{array}{l}\text { Bartonella spp. nuoG } \\
\text { qPCR-positive samples }\end{array}$} & \multirow[b]{2}{*}{$\begin{array}{l}C q \text { mean } \\
\text { values }\end{array}$} & \multirow[b]{2}{*}{$\begin{array}{l}\text { Quantification } \\
\text { (nuoG-copies } \mu \mathrm{L}^{-1} \text { ) }\end{array}$} & \multirow[b]{2}{*}{$\begin{array}{l}\text { gltA } \\
\text { cPCR }\end{array}$} & \multicolumn{3}{|c|}{ Closest BLAST identity (\%) } \\
\hline & & & & Organism & Identity & $\begin{array}{l}\text { Accession } \\
\text { number }\end{array}$ \\
\hline A6B & NA & NA & Positive NS & - & - & - \\
\hline A1C & NA & $\mathrm{NA}$ & Negative & - & - & - \\
\hline $\mathrm{A} 3 \mathrm{C}$ & $28 \cdot 18$ & $6 \cdot 22 \times 10^{2}$ & Positive & Bartonella henselae & $(100 \%)$ & HG965802.1 \\
\hline A5C & $30 \cdot 85$ & $2 \cdot 22 \times 10^{2}$ & Positive & Bartonella henselae & $(100 \%)$ & HG965802.1 \\
\hline A7C & NA & NA & Negative & - & - & - \\
\hline A1E & NA & NA & Positive & $\begin{array}{l}\text { Bartonella } \\
\text { clarridgeiae }\end{array}$ & $(100 \%)$ & FN645454.1 \\
\hline A8E & NA & NA & Positive NS & - & - & - \\
\hline $\mathrm{A} 10 \mathrm{E}$ & $29 \cdot 72$ & $2 \cdot 47 \times 10^{3}$ & Positive & $\begin{array}{l}\text { Bartonella } \\
\text { clarridgeiae }\end{array}$ & $(100 \%)$ & FN645454.1 \\
\hline A10F & NA & NA & Negative & - & - & - \\
\hline A10G & NA & NA & Positive & Bartonella henselae & $(100 \%)$ & HG965802.1 \\
\hline A5I & NA & NA & Negative & - & - & - \\
\hline B10A & $26 \cdot 70$ & $4 \cdot 08 \times 10^{3}$ & Positive & $\begin{array}{l}\text { Bartonella } \\
\text { clarridgeiae }\end{array}$ & $(100 \%)$ & KJ170236.1 \\
\hline $\mathrm{B} 4 \mathrm{C}$ & $30 \cdot 24$ & $4 \cdot 34 \times 10^{2}$ & Positive & Bartonella henselae & $(100 \%)$ & HG965802.1 \\
\hline $\mathrm{B} 5 \mathrm{C}$ & $27 \cdot 72$ & $2 \cdot 24 \times 10^{3}$ & Positive & $\begin{array}{l}\text { Bartonella } \\
\text { clarridgeiae }\end{array}$ & $(100 \%)$ & FN645454.1 \\
\hline $\mathrm{B} 6 \mathrm{C}$ & NA & $4 \cdot 42 \times 10^{2}$ & Positive & $\begin{array}{l}\text { Bartonella } \\
\text { clarridgeiae }\end{array}$ & $(100 \%)$ & FN645454.1 \\
\hline $\mathrm{B} 7 \mathrm{C}$ & NA & NA & Positive & Bartonella khoelerae & $(100 \%)$ & AF176091.1 \\
\hline $\mathrm{B} 2 \mathrm{D}$ & $26 \cdot 66$ & $4.85 \times 10^{2}$ & Positive & Bartonella henselae & $(100 \%)$ & HG965802.1 \\
\hline $\mathrm{B} 4 \mathrm{D}$ & $30 \cdot 24$ & $8 \cdot 0 \times 10^{2}$ & Positive & Bartonella henselae & $(100 \%)$ & HG965802.1 \\
\hline B1E & $31 \cdot 25$ & $5 \cdot 46 \times 10^{0}$ & Positive & Bartonella henselae & $(99 \%)$ & HG965802.1 \\
\hline B7E & $29 \cdot 47$ & $7 \cdot 35 \times 10^{1}$ & Positive & Bartonella henselae & $(99 \%)$ & HG965802.1 \\
\hline $\mathrm{B} 1 \mathrm{~F}$ & $31 \cdot 20$ & $5 \cdot 78 \times 10^{0}$ & Positive & Bartonella henselae & $(99 \%)$ & HG965802.1 \\
\hline $\mathrm{B} 2 \mathrm{~F}$ & $30 \cdot 10$ & $2 \cdot 11 \times 10^{2}$ & Positive NS & - & - & - \\
\hline B3F & $21 \cdot 80$ & $3 \cdot 19 \times 10^{4}$ & Positive NS & - & - & - \\
\hline $\mathrm{B} 4 \mathrm{~F}$ & $32 \cdot 12$ & $3 \cdot 15 \times 10^{1}$ & Positive NS & - & - & - \\
\hline $\mathrm{B} 5 \mathrm{~F}$ & $31 \cdot 59$ & $4 \cdot 49 \times 10^{1}$ & Positive & $\begin{array}{l}\text { Bartonella } \\
\text { clarridgeiae }\end{array}$ & $(100 \%)$ & FN645454.1 \\
\hline B6F & $27 \cdot 22$ & $8 \cdot 14 \times 10^{2}$ & Positive & Bartonella henselae & $(100 \%)$ & HG965802.1 \\
\hline $\mathrm{B} 7 \mathrm{~F}$ & $32 \cdot 23$ & $2 \cdot 19 \times 10^{1}$ & Positive & Bartonella henselae & $(100 \%)$ & HG965802.1 \\
\hline B9F & NA & NA & Negative & - & - & - \\
\hline B2G & $34 \cdot 88$ & $2 \cdot 13 \times 10^{0}$ & Positive NS & - & - & - \\
\hline B3G & NA & NA & Positive & Bartonella henselae & $(100 \%)$ & HG965802.1 \\
\hline B4G & NA & NA & Negative & - & - & - \\
\hline B5G & NA & NA & Positive & Bartonella henselae & $(99 \%)$ & HG965802.1 \\
\hline $\mathrm{B} 5 \mathrm{H}$ & $27 \cdot 61$ & $1 \cdot 14 \times 10^{3}$ & Positive & $\begin{array}{l}\text { Bartonella } \\
\text { clarridgeiae }\end{array}$ & $(100 \%)$ & FN645454.1 \\
\hline $\mathrm{B} 8 \mathrm{H}$ & $31 \cdot 37$ & $9 \cdot 56 \times 10^{1}$ & Positive & Bartonella henselae & $(100 \%)$ & HG969191.1 \\
\hline B9H & $35 \cdot 85$ & $5 \cdot 05 \times 10^{0}$ & Negative & - & - & - \\
\hline $\mathrm{B} 10 \mathrm{H}$ & $29 \cdot 12$ & $4 \cdot 24 \times 10^{2}$ & Positive & Bartonella henselae & $(100 \%)$ & HG969191.1 \\
\hline B2I & NA & $\mathrm{NA}$ & Positive NS & - & - & - \\
\hline C6A & $33 \cdot 16$ & $2.93 \times 10^{1}$ & Positive NS & - & - & - \\
\hline C9A & NA & $\mathrm{NA}$ & Positive NS & - & - & - \\
\hline $\mathrm{C} 6 \mathrm{~B}$ & NA & NA & Negative & - & - & - \\
\hline $\mathrm{C} 7 \mathrm{~B}$ & NA & NA & Negative & - & - & - \\
\hline $\mathrm{C} 8 \mathrm{~B}$ & $34 \cdot 06$ & $2.54 \times 10^{1}$ & Positive NS & - & - & - \\
\hline $\mathrm{C} 2 \mathrm{C}$ & $29 \cdot 12$ & $2 \cdot 51 \times 10^{2}$ & Negative & - & - & - \\
\hline $\mathrm{C} 3 \mathrm{C}$ & $25 \cdot 65$ & $2 \cdot 57 \times 10^{3}$ & Negative & - & - & - \\
\hline $\mathrm{C} 2 \mathrm{D}$ & $29 \cdot 33$ & $3.95 \times 10^{1}$ & Positive & Bartonella henselae & $(99 \%)$ & HG965802.1 \\
\hline $\mathrm{C} 1 \mathrm{E}$ & NA & NA & Positive NS & - & - & - \\
\hline $\mathrm{C} 2 \mathrm{E}$ & NA & NA & Positive NS & - & - & - \\
\hline C3E & NA & NA & Positive & Bartonella henselae & $(99 \%)$ & HG965802.1 \\
\hline $\mathrm{C} 4 \mathrm{E}$ & NA & $\mathrm{NA}$ & Positive NS & - & - & - \\
\hline C5E & $32 \cdot 28$ & $4.52 \times 10^{0}$ & Positive NS & - & - & - \\
\hline $\mathrm{C} 9 \mathrm{~F}$ & NA & $\mathrm{NA}$ & Positive & Bartonella henselae & $(100 \%)$ & HG965802.1 \\
\hline $\mathrm{C} 2 \mathrm{G}$ & NA & $\mathrm{NA}$ & Negative & - & - & - \\
\hline
\end{tabular}


Table 1. (Cont.)

\begin{tabular}{|c|c|c|c|c|c|c|}
\hline \multirow[b]{2}{*}{$\begin{array}{l}\text { Bartonella spp. nuoG } \\
\text { qPCR-positive samples }\end{array}$} & \multirow[b]{2}{*}{$\begin{array}{l}C q \text { mean } \\
\text { values }\end{array}$} & \multirow[b]{2}{*}{$\begin{array}{l}\text { Quantification } \\
\text { (nuoG-copies } \mu \mathrm{L}^{-1} \text { ) }\end{array}$} & \multirow[b]{2}{*}{$\begin{array}{l}\text { gltA } \\
\text { cPCR }\end{array}$} & \multicolumn{3}{|c|}{ Closest BLAST identity (\%) } \\
\hline & & & & Organism & Identity & $\begin{array}{l}\text { Accession } \\
\text { number }\end{array}$ \\
\hline $\mathrm{C} 7 \mathrm{G}$ & NA & NA & Negative & - & - & - \\
\hline $\mathrm{C} 8 \mathrm{G}$ & $29 \cdot 51$ & $2 \cdot 88 \times 10^{2}$ & Positive & $\begin{array}{l}\text { Bartonella } \\
\text { clarridgeiae }\end{array}$ & $(100 \%)$ & FN645454.1 \\
\hline $\mathrm{C} 5 \mathrm{H}$ & NA & NA & Positive NS & - & - & - \\
\hline $\mathrm{C} 1 \mathrm{~J}$ & $32 \cdot 04$ & $1 \cdot 61 \times 10^{1}$ & Positive & $\begin{array}{l}\text { Bartonella } \\
\text { clarridgeiae }\end{array}$ & $(100 \%)$ & FN645454.1 \\
\hline $\mathrm{C} 2 \mathrm{~J}$ & $26 \cdot 32$ & $8 \cdot 25 \times 10^{2}$ & Positive & - & - & - \\
\hline D9A & $26 \cdot 91$ & $5 \cdot 44 \times 10^{2}$ & Positive & $\begin{array}{l}\text { Bartonella } \\
\text { clarridgeiae }\end{array}$ & $(100 \%)$ & FN645454.1 \\
\hline D6B & $32 \cdot 27$ & $5.67 \times 10^{0}$ & Negative & - & - & - \\
\hline D3D & $33 \cdot 05$ & $3 \cdot 51 \times 10^{0}$ & Negative & - & - & - \\
\hline D6D & $30 \cdot 72$ & $2 \cdot 47 \times 10^{2}$ & Positive NS & - & - & - \\
\hline D8D & $35 \cdot 08$ & $1.66 \times 10^{1}$ & Negative & - & - & - \\
\hline $\mathrm{D} 8 \mathrm{E}$ & $32 \cdot 11$ & $1.04 \times 10^{2}$ & Positive NS & - & - & - \\
\hline $\mathrm{D} 1 \mathrm{H}$ & NA & NA & Negative & - & - & - \\
\hline $\mathrm{D} 2 \mathrm{H}$ & NA & NA & Positive NS & - & - & - \\
\hline D4H & NA & NA & Negative & - & - & - \\
\hline D6H & $30 \cdot 19$ & $1.13 \times 10^{2}$ & Positive NS & - & _- & - \\
\hline
\end{tabular}

cPCR, conventional PCR; NS, cPCR-positive sample but with a weak band intensity, which precluded sequencing; NA, data not available due to inconsistent results.

Twenty glt $A$-cPCR-positive samples presented weak band intensity, precluding sequencing. BLAST and phylogenetic analyses supported the identification of $62.0 \%(18 / 29)$ as $B$. henselae, $34 \cdot 4 \%$ as B. clarridgeiae $(10 / 29)$ and $3 \cdot 4 \%(1 / 29)$ as B. koehlerae (Table 1).

Analysis of 14 sequenced products based on the glt $A$ gene (GenBank accession numbers KX024499, $\mathrm{KX} 024500$, KX024503, KX024505, KX024509KX024513， KX024515-KX024518， KX024520 KX024524) showed 99-100\% identicalness with $B$. henselae (GenBank accession numbers HG965802; KJ170236). These fragments were positioned close to other $B$. henselae isolates, supported by high bootstrap values (96) in maximum-likelihood phylogeneitc analysis. Additionally, the analysis of six sequenced products based on the glt $A$ region (GenBank accession numbers KX024501, KX024502, KX024504, KX024506, KX024507, KX024514, KX024519, KX024525-KX024527) showed 100\% sequence identity with B. clarridgeiae (GenBank accession number FN645454 and KJ170236), and clustering with other B. clarridgeiae isolates. Finally, the analysis of one sequenced product based on the glt $A$ region (GenBank accession number KX024508) showed $100 \%$ identicalness with B. koehlerae (GenBank accession number AF176091) and was positioned close to the American isolate (Fig. 2).

\section{Nucleotide diversity}

Overall, the Bartonella species identified in the present study had a low genetic diversity. Of the
$18 B$. henselae glt $A$ sequences analysed, only three different haplotypes were identified. The haplotype number \#Bh1 with ten sequences was the most abundant haplotype, haplotypes \# Bh2 and \# Bh3 contained two and six sequences, respectively. Additionally, only two haplotypes were identified in the $B$. clarridgeiae sequences. While nine sequences formed the haplotype number \#Bc1, only one sequence formed the haplotype number \#Bc2. Both Bartonella species had a low number of variable sites, haplotypes and nucleotide diversity (Table 2).

\section{Haematological analysis}

All variables were non-normally distributed. The only haematological parameter that was significantly different was the MCV $(P<0 \cdot 0001)$, which was significantly lower in Bartonella-positive cats (mean $=41.5 \mathrm{fL})$ than in Bartonella-negative ones $($ mean $=44 \mathrm{fL})$. There were no other significant differences between Bartonella-positive and -negative cats.

\section{DISCUSSION}

Cats are the major hosts of $B$. henselae, B. clarridgeiae and $B$. koehlerae (Mogollon-Pasapera et al. 2009). Since the pathogens can be transferred to humans through scratches or bites, public monitoring of its prevalence in cats is important (Breitschwerdt, 2008). This is the first study to investigate Bartonella spp. molecular prevalence in 


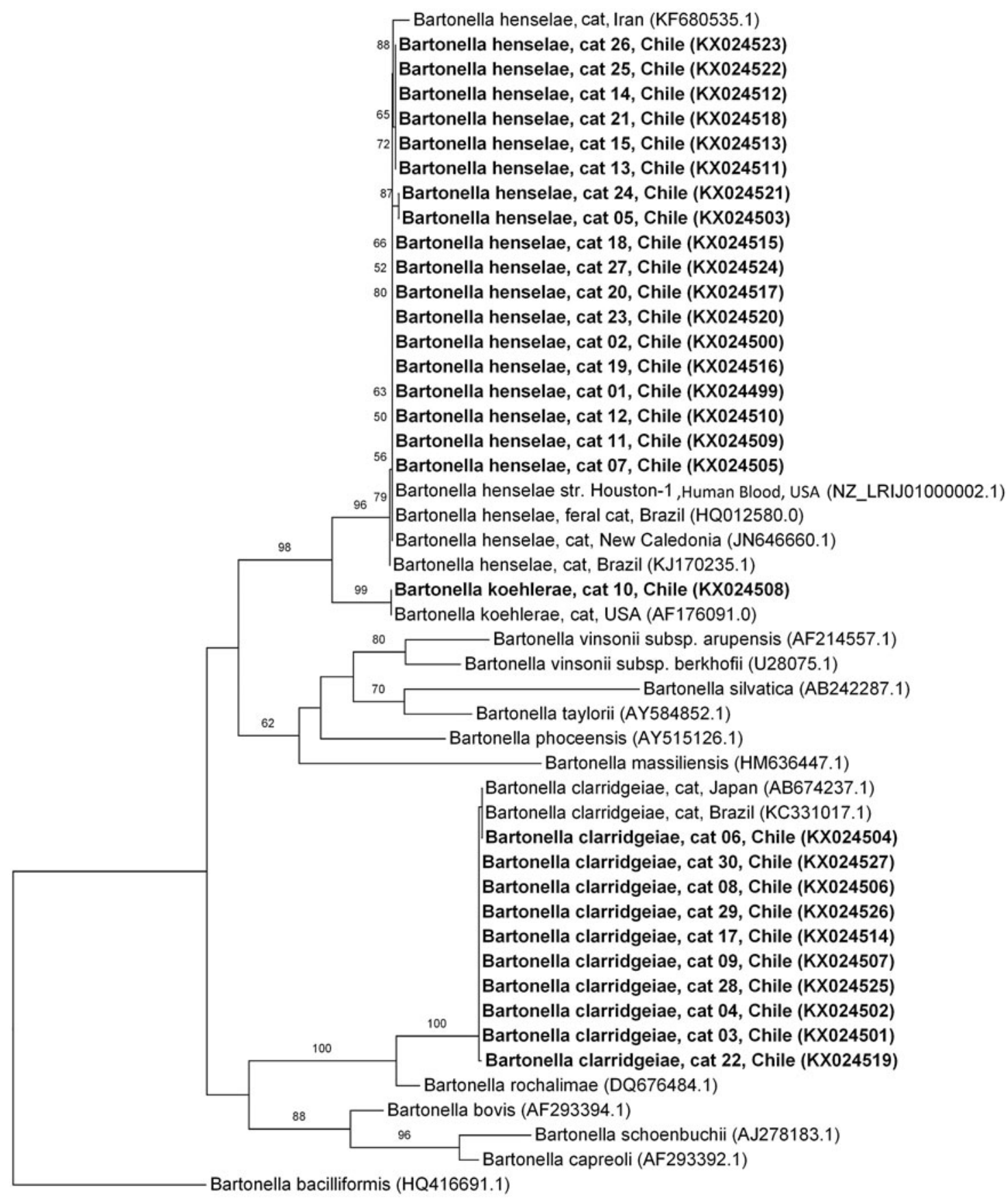

Fig. 2. Phylogenetic relationships within the Bartonella genus based on a $680 \mathrm{bp}$ fragment of the gltA gene. The tree was inferred by using the ML method and evolutive model GAMMA GTR + I. The sequences detected in the present study are bold highlighted. The numbers at the nodes correspond to bootstrap values higher than $50 \%$ obtained with 1000 replicates. Bartonella bacilliformis was used as an outgroup.

Table 2. Polymorphism and genetic diversity of gltA Bartonella sequences detected in cats from Valdivia, Chile

\begin{tabular}{lllllllll}
\hline \hline Species & $(\mathrm{bp})$ & $N$ & $\mathrm{VS}$ & $\mathrm{GC}(\%)$ & $h$ & $h d$ (mean \pm s.D.) & $\pi$ (mean \pm s.D.) & $K$ \\
\hline Bartonella henselae & 674 & 18 & 3 & $37 \cdot 1$ & 3 & $0 \cdot 601 \pm 0 \cdot 080$ & $0 \cdot 001 \pm 0 \cdot 0003$ & $0 \cdot 888$ \\
Bartonella clarridgeiae & 674 & 10 & 1 & $38 \cdot 2$ & 2 & $0 \cdot 200 \pm 0 \cdot 154$ & $0 \cdot 0003 \pm 0 \cdot 0002$ & $0 \cdot 200$ \\
\hline \hline
\end{tabular}

$N$, number of sequences analysed; VS, number of variable sites; GC, G + C content; $h$, number of haplotypes; $h d$, haplotypes diversity; S.D., standard deviation; $\pi$, nucleotide diversity (per site $=\mathrm{PI}$ ); $K$, average number of nucleotide difference. 
a population of domestic cats from southern Chile. Previous reports in Chile described high seroprevalence of B. henselae in Valdivia (Zaror et al. 2002; Ferrés et al. 2006a), Santiago and Coquimbo cities (Ferrés et al. 2005). Identification of $B$. henselae in solid cultures from cat $(25 / 60)$ blood samples was performed in central Chile (Santiago City) (Ferrés et al. 2005).

Only few studies in South America have evaluated the molecular occurrence of Bartonella spp. in cats, mostly carried in Brazil (Staggemeier et al. 2010; Crissiuma et al. 2011; Braga et al. 2012; de Bortoli et al. 2012; Miceli et al. 2013). Cats from Valdivia, Southern Chile, showed a similar prevalence $(18 \cdot 1 \%)$ to the one described in Buenos Aires, Argentina (17.8\%) (Cicuttin et al. 2014) and Southern Brazil (17·0\%) (Staggemeier et al. 2010), lower than that observed in cats from Galapagos Island, Ecuador (44.0\%) (Levy et al. 2008) and higher than central-western (2.2\%) (Miceli et al. 2013), northeastern (4.5\%) (Braga et al. 2012) and southeastern (4.3\%) (de Bortoli et al. 2012) Brazil. However, comparison is difficult, because of the low number of cats and inclusion criteria used in some studies. In general, a higher prevalence was observed in stray cats (as high as 61.1\%) (Boulouis et al. 2005; Gutiérrez et al. 2013), young adult cats living in shelters (36\%) (Fleischman et al. 2015) and cats from spaying/neutering program (42.5\%) (Crissiuma et al. 2011). Differences in prevalence may reflect variations in the groups of studied cats, or by geographical variations, such as climate and bloodsucking arthropod distribution, where the presence of fleas (Boulouis et al. 2005) is a risk factor for Bartonella infection. Furthermore, a direct comparison between studies is difficult because of the differences in $\mathrm{cPCR}$ and qPCR diagnostic assays (André et al. 2016).

As described worldwide (Chomel and Kasten, 2010), B. henselae was the most prevalent species in Southern Chile, followed by $B$. clarridgeiae. The latter was less frequently isolated from domestic cats than $B$. henselae, as it appears to be difficult to isolate and is unevenly distributed in cat populations (Chomel et al. 2004). Only one sampled cat was positive for B. koehlerae, which has rarely been detected in domestic cats worldwide (Avidor et al. 2004; Chomel and Kasten, 2010; Fleischman et al. 2015). To the best of our knowledge, B. koehlerae is detected for the first time in cats from South America.

The low genetic diversity of Bartonella species identified in cats from Southern Chile is in accordance with the high intra species similarity between glt $A$ gene sequences of various Bartonella spp. (99.80-100\%) (Birtles and Raoult, 1996). Since $<1 \cdot 00 \%$ genomic variety exists between various strains of $B$. henselae, a low diversity is a common finding (Guy et al. 2012). Due to the low genetic diversity, each Bartonella species from Chile clustered together and with other Bartonella spp. described in cats from Brazil, Iran and USA (Droz et al. 1999; André et al. 2014; Fard et al. 2016).

Bartonella henselae isolates clustered with both Houston-1 and Marseille strains, presenting a high similarity with these. Since Multi Locus Sequence Typing was not performed in the present study, it was not possible to determine which strains are circulating in Chilean cats. Most human cases of CSD are caused by $B$. henselae type Houston-1, suggesting that type Houston-1 strains could be more virulent to humans (Boulouis et al. 2005) than Marseille (type II), which is more frequently identified in cats (Chomel et al. 2004; Boulouis et al. 2005). Bartonella henselae-type Marseille is the dominant type in cat populations from Western Europe (France, Germany, Italy, The Netherlands and UK) and Australia (Boulouis et al. 2005), whereas type Houston-1 is more frequently reported in human cases in the same regions (Arvand et al. 2007). Houston-1 is more frequent in cats from Asia (Japan and the Philippines) (Boulouis et al. 2005). In North America (USA), type II is more prevalent in cats on the West Coast (California) (Chomel et al. 1995; Chang et al. 2002; Fleischman et al. 2015) but a 50-50\% (types I and II) was described on the East Coast (North Carolina and Florida) (Guptil et al. 2004). Bartonella henselae isolates obtained from cats in Guatemala (Bai et al. 2015) and Argentina (Cicuttin et al. 2014) were Houston type I group, suggesting that it could be the major genotype in Central and South America. Nevertheless, more studies on $B$. henselae diversity in other countries, including Chile, are needed to prove this hypothesis.

The mean number of Bartonella spp. nuoGcopies $\mu \mathrm{L}^{-1}$ in cats from southern Chile was lower than that described in naturally infected cats from Brazil, using the same qPCR protocol (André et al. 2016). Indeed, low initial DNA copies in some blood samples from Chilean cats could produce inconsistent quantification results in the Bartonella-qPCR assay, represented by the Monte Carlo effect (Bustin et al. 2009). The low number of nuoG-Bartonella copies in blood samples from cats in our study may be explained by the characteristics of Bartonella spp. infection. After infecting their hosts, Bartonella may cause a persistent bacteraemia in cats, which is undetectable (Breitschwerdt et al. 2010a). Long-term intraerythrocytic bacteraemia in reservoir mammals is frequently described and represents a common strategy of Bartonella for achieving infection without producing organ damage, generating only chronic, asymptomatic, infection (Chomel et al. 2009).

A lower MCV, within the reference values, was the only haematological finding in Bartonella-positive cats and was not considered clinically relevant. 
As observed in Chilean cats, haematological abnormalities are rarely described in naturally infected cats that seem to be healthy carriers of the bacterium (Boulouis et al. 2005; Chomel et al. 2009). In neotropical felids haematological abnormalities were not associated with Bartonella spp. natural infection (Guimaraes et al. 2010). On the contrary, eosinophilia (Kordick et al. 1999) and neutrophilia (Guptill et al. 1997) were observed in experimentally infected cats. It is important to state that in experimentally infected cats, usually the inoculum dose was very high (Guptill et al. 1997). Furthermore, strain variability among $B$. henselae isolates may contribute to enhanced pathogenicity in experimentally infected cats (O'Reilly et al. 1999).

Circulation of the three Bartonella species in Valdivia cats strengthens the importance of the feline population as a source of zoonotic agents and represents a potential infection risk to humans. While most cats are asymptomatic after becoming infected with $B$. henselae, they serve as reservoirs of the agent and may transmit the infection to humans (Breitschwerdt et al. 2010a). Data on CSD prevalence in Chile reports a $10.3 \%$ infection rate with $B$. henselae in children from Central Chile (Ferrés et al. 2006b). Also, asymptomatic catowners from southern Chile showed serological exposure (18\%) to B. henselae (Zaror et al. 2002) and a high seroprevalence $(60 \%)$ was observed in humans with an occupational risk in the Bío Bío region, Chile (Troncoso et al. 2016). The presence of $B$. clarridgeiae and B. koehlerae in cats suggests the need to consider these agents when testing clinical samples from suspected human cases in Chile, along with $B$. henselae.

\section{Concluding remarks}

The overall prevalence of Bartonella spp. in domestic cats from Valdivia, Southern Chile, is in accordance with that previously described in South America. Bartonella-positive cats had low DNA bacterial loads and their haematological parameters varied minimally. Low genetic diversity was reported among the $B$. henselae and $B$. clarridgeiae haplotypes in the present study. Three Bartonella species circulate in the studied cat population of Valdivia. Bartonella clarridgeiae is reported for the first time in cats from Chile and B. koehlerae in cats from South America.

\section{ACKNOWLEDGEMENTS}

We thank the veterinary team of the UACh Veterinary Hospital for their helpful contributions in collecting samples. We also wish to thank the team of the Inmunoparasitology Laboratory, Faculdade de Ciencias Agárias e Veterinarias of the Universidade Estadual Paulista, Jaboticabal, Brazil, for their technical support.
FINANCIAL SUPPORT

This work was supported by Beca Santander Iberoamérica Jovenes Investigadores, 2015, Chile.

\section{CONFLICT OF INTEREST}

The authors do not have any conflicts of interest to declare.

\section{REFERENCES}

André, M. R., Baccarim Denardi, N.C., Marques de Sousa, K. C., Gonçalves, L. R., Henrique, P.C., Grosse Rossi Ontivero, C. R., Lima Gonzalez, I. H., Cabral Nery, C. V., Fernandes Chagas, C. R., Monticelli, C., Alexandre de Santis, A.C. G. and Machado, R. Z. (2014). Arthropod-borne pathogens circulating in free-roaming domestic cats in a zoo environment in Brazil. Ticks and Tick-borne Diseases 5, $545-551$.

André, M. R., Dumler, J. S., Herrera, H. M., Goncalves, L. R., de Sousa, K. C., Scorpio, D. G., de Santis, A. C. G. A., Domingos, I. H., de Macedo, G. C. and Machado, R. Z. (2016). Assessment of a quantitative $5^{\prime}$ nuclease real-time polymerase chain reaction using the nicotinamide adenine dinucleotide dehydrogenase gamma subunit (nuoG) for Bartonella species in domiciled and stray cats in Brazil. Fournal of Feline Medicine and Surgery 18, 783-790.

Arvand, M., Feil, E. J., Giladi, M., Boulouis, H. and Viezens, J. (2007). Multi-locus sequence typing of Bartonella henselae isolates from three continents reveals hypervirulent and feline-associated clones. PLoS ONE 2, e1346.

Avidor, B., Graidy, M., Efrat, G., Leibowitz, C., Shapira, G., Schattner, A., Zimhony, O. and Giladi, M. (2004). Bartonella koehlerae, a new cat-associated agent of culture-negative human endocarditis. Fournal of Clinical Microbiology 42, 3462-3468.

Bai, Y., Rizzo, M.F., Alvarez, D., Moran, D., Peruski, L.F. and Kosoy, M. (2015). Coexistence of Bartonella henselae and B. clarridgeiae in populations of cats and their fleas in Guatemala. Fournal of Vector Ecology 40, 327-332

Bergh, K., Bevanger, L., Hanssen, I. and Loseth, K. (2002). Low prevalence of Bartonella henselae infections in Norwegian domestic and feral cats. APMIS 110, 309-314.

Billeter, S. A., Gundi, V. A. K. B., Rood, M. P. and Kosoy, M. Y. (2011) Molecular detection and identification of Bartonella species in Xenopsylla cheopis fleas (Siphonaptera: Pulicidae) collected from Rattus norvegicus rats in Los Angeles, California. Applied and Environmental Microbiology 77, 7850-7852.

Birtles, R. J. and Raoult, D. (1996). Comparison of partial citrate synthase gene (gltA) sequences for phylogenetic analysis of Bartonella species. International Fournal of Systematic Bacteriology 46, 891-897.

Boulouis, H.-J., Chao-chin, C., Henn, J. B., Kasten, R. W. and Chomel, B. B. (2005). Factors associated with the rapid emergence of zoonotic Bartonella infections. Veterinary Research 36, 383-410.

Braga, M. do S. C. de O., de Paiva Diniz Diniz, P. P. V., André, M. R., de Bortoli, C. P. and Machado, R. Z. (2012). Molecular characterisation of Bartonella species in cats from São Luís, state of Maranhão, northeastern Brazil. Memorias do Instituto Oswaldo Cruz 107, 772-777.

Breitschwerdt, E. B. (2008). Feline bartonellosis and cat scratch disease. Veterinary Immunology and Immunopathology 123, 167-171.

Breitschwerdt, E. B., Maggi, R. G., Mozayeni, B. R., Hegarty, B. C., Bradley, J. M. and Mascarelli, P.E. (2010a). PCR amplification of Bartonella koehlerae from human blood and enrichment blood cultures. Parasites and Vectors 3, 1-9.

Breitschwerdt, E. B., Maggi, R. G., Chomel, B. B. and Lappin, M. R. (2010b). Bartonellosis: an emerging infectious disease of zoonotic importance to animals and human beings. Fournal of Veterinary Emergency and Critical Care 20, 8-30.

Bustin, S. A., Benes, V., Garson, J. A., Hellemans, J., Huggett, J., Kubista, M., Mueller, R., Nolan, T., Pfaff, M. W. and Shipley, G. L. (2009). Special report the MIQE guidelines: minimum information for publication of quantitative real-time PCR experiments. Clinical Chemistry 55, 611-622.

Chang, C., Chomel, B. B., Kasten, R. W., Tappero, J. W., Sanchez, M. A. and Koehler, J. E. (2002). Molecular epidemiology of Bartonella henselae infection in human immunodeficiency virus-infected patients and their cat contacts, using pulsed-field gel electrophoresis and genotyping. Fournal of Infectious Diseases 186, 1733-1739. 
Chomel, B. B. and Kasten, R. W. (2010). Bartonellosis, an increasingly recognized zoonosis. Fournal of applied Mycrobiology 109, 743-750.

Chomel, B. B., Abbott, R. C., Kasten, R. W., Floyd-Hawkins, K. A., Kass, P. H., Glaser, C. A., Pedersen, N. C. and Koehler, J. E. (1995). Bartonella henselae prevalence in domestic cats in California: risk factors and association between bacteremia and antibody titers. Fournal of Clinical Microbiology 33, 2445-2450.

Chomel, B. B., Carlos, E. T., Kasten, R. W., Yamamoto, K., Chang, C. C., Carlos, R. S., Abenes, M. V. and Pajares, C. M. (1999). Bartonella henselae and Bartonella clarridgeiae infection in domestic cats from The Philippines. American Fournal of Tropical Medicine and Hygiene 60 593-597.

Chomel, B. B., Boulouis, H. J. and Breitschwerdt, E. B. (2004). Cat scratch disease and other zoonotic Bartonella infections. Fournal of the American Veterinary Medical Association 224, 1270-1279.

Chomel, B. B., Boulouis, H.-J., Breitschwerdt, E. B., Kasten, R. W., Vayssier-Taussat, M., Birtles, R. J., Koehler, J. E. and Dehio, C. (2009). Ecological fitness and strategies of adaptation of Bartonella species to their hosts and vectors. Veterinary Research 40, 29.

Cicuttin, G. L., Brambati, D. F., De Gennaro, M. F., Carmona, F., Isturiz, M. L., Pujol, L. E., Belerenian, G. C. and Gil, H. (2014). Bartonella spp. in cats from Buenos Aires, Argentina. Veterinary Microbiology 168, 225-228.

Crissiuma, A., Favacho, A., Gershony, L., Mendes-de-Almeida, F., Gomes, R., Rozental, T., Barreira, J., Lemos, E. and Labarthe, N. (2011). Prevalence of Bartonella species DNA and antibodies in cats (Felis catus) submitted to a spay/neuter program in Rio de Janeiro, Brazil. Fournal of Feline Medicine and Surgery 13, 149-151.

de Bortoli, C., André, M., Seki, M., Pinto, A., Machado, S. Z. and Machado, R.Z. (2012). Detection of hemoplasma and Bartonella species and co-infection with retroviruses in cats subjected to a spaying/neutering program in Jaboticabal, SP, Brazil. Revista Brasileira de Parasitologia Veterinaria 21, 219-223.

Droz, S., Chi, B., Horn, E., Steigerwalt, A. G., Whitney, A. M. and Brenner, D. J. (1999). Bartonella koehlerae sp. nov., isolated from cats. Fournal of Clinical Microbiology 37, 1117-1122.

Fard, M. R. N., Vahedi, S. M., Ashrafi, I., Alipour, F., Sharafi, G., Akbarein, H. and Aldavood, S. J. (2016). Molecular identification and phylogenic analysis of Bartonella henselae isolated from Iranian cats based on glt A gene. Veterinary Research Forum 7, 69-72.

Fenollar, F. and Raoult, D. (2004). Molecular genetic methods for the diagnosis of fastidious microorganisms. APMIS 112, 785-807.

Ferrés, G. M., Abarca, V.K., Prado, D.P., Montecinos, P. L. Navarrete, C. M. and Vial, C. P. A. (2006a). Prevalencia de anticuerpos contra Bartonella henselae en niños, en adolescentes y en una población de riesgo ocupacional en Chile. Revista médica de Chile 134, 863-867.

Ferrés, M., Abarca, K., Godoy, P., García, P., Palavecino, E., Méndez, G., Valdés, A., Ernst, S., Thibaut, J., Koberg, J., Chanqueo, L. and Vial, P. A. (2005). Presence of Bartonella henselae in cats: natural reservoir quantification and human exposition risk of this zoonoses in Chile. Revista médica de Chile 133, 1465-1471.

Ferrés, M., Abarca, V. K., Prado, D. P., Montecinos, P. L. Navarrete, C. M. and Vial, C. P. A. (2006b). Prevalencia de anticuerpos contra Bartonella henselae en niños, en adolescentes y en una población de riesgo ocupacional en Chile. Revista médica de Chile 134, 863-867.

Fleischman, D. A., Chomel, B. B., Kasten, R. W., Stuckey, M. J., Scarlet, J., Liu, H., Boulouis, H., Haddad, N. and Pedersen, N. (2015). Bartonella infection among cats adopted from a San Francisco Shelter, revisited. Applied and Environmental Microbiology 81, 6446-6450. Guimaraes, A. M. S., Brandão, P. E., Moraes, W., Kiihl, S., Santos, L. C., Filoni, C., Cubas, Z.S., Robes, R. R., Marques, L. M., Neto, R. L., Yamaguti, M., Oliveira, R. C., Catão-Dias, J. L., Richtzenhain, L. J., Messick, J. B., Biondo, A. W. and Timenetsky, J. (2010). Detection of Bartonella spp. in neotropical felids and evaluation of risk factors and hematological abnormalities associated with infection. Veterinary Microbiology 142, 346-351.

Guptill, L., Wu, C., Hogenesch, H., Slater, L., Glickman, N., Dunham, A., Syme, H. and Glickman, L. (2004). Prevalence, risk factors, and genetic diversity of Bartonella henselae infections in pet cats in four regions of the United States. Fournal of Clinical Microbiology 42, 652-659.

Guptill, L., Slater, L., Wu, C.C., Lin, T.L., Glickman, L. T., Welch, D. F. and HogenEsch, H. (1997). Experimental infection of young specific pathogen-free cats with Bartonella henselae. Fournal of Infectious Diseases 176, 206-216.
Gutiérrez, R., Morick, D., Gross, I., Winkler, R., Abdeen, Z. and Harrus, S. (2013). Bartonellae in domestic and stray cats from Israel: comparison of bacterial cultures and high-resolution melt real-time PCR as diagnostic methods. Vector-Borne and Zoonotic Diseases 13, 857-864.

Guy, L., Nystedt, B., Sun, Y., Näslund, K., Berglund, E. C. and Andersson, S. G. E. (2012). A genome-wide study of recombination rate variation in Bartonella henselae. BMC evolutionary Biology 12, 65.

Helps, C., Lait, P., Damhuis, A., Björnehammar, U., Bolta, D., Brovida, C., Chabanne, L., Egberink, H., Ferrand, G., Fontbonne, A., Pennisi, M. G., Gruffydd-Jones, T., Gunn-Moore, D., Hartmann, K., Lutz, H., Malandain, E., Möstl, K., Stengel, C., Harbour, D. A. and Graat, E. A. M. (2005). Factors associated with upper respiratory tract disease caused by feline herpesvirus, feline calicivirus, Chlamydophila felis and Bordetella bronchiseptica in cats: experience from 218 European catteries. Veterinary Record 156, 669-673.

Kordick, D. L., Brown, T. T., Shin, K. and Breitschwerdt, E. B. (1999). Clinical and pathologic evaluation of chronic Bartonella henselae or Bartonella clarridgeiae infection in cats. Fournal of Clinical Microbiology 37, 1536-1547.

Levy, J. K., Crawford, P. C., Lappin, M. R., Dubovi, E. J., Levy, M. G., Alleman, R., Tucker, S. J. and Clifford, E. L. (2008). Infec tious diseases of dogs and cats on Isabel a Island, Galapagos. Fournal of Veterinary Internal Medicine 22, 60-65.

Librado, P. and Rozas, J. (2009). DnaSP v5: a software for comprehensive analysis of DNA polymorphism data. Bioinformathics 25 , 1451-1452.

Maggi, R. G., Duncan, A. W. and Breitschwerdt, E. B. (2005). Novel chemically modified liquid medium that will support the growth of seven Bartonella species. Fournal of Clinical Microbiology 43, 2651-2655.

Maggi, R. G., Mascarelli, P.E., Pultorak, E. L., Hegarty, B. C., Bradley, J. M., Mozayeni, B. R. and Breitschwerdt, E. B. (2011). Bartonella spp. bacteremia in high-risk immunocompetent patients. Diagnostic Microbiology and Infectious Disease 71, 430-437.

Miceli, N. G., Gavioli, F. A., Gonçalves, L. R., André, M. R., Souza, V., Marques de Sousa, K. C. and Machado, R. Z. (2013). Molecular detection of feline arthropod-borne pathogens in cats in Cuiabá, state of Mato Grosso, central-western region of Brazil. Rev Bras Parasitol Vet 22, 385-390.

Miller, M. A., Pfeiffer, W. and Schwartz, T. (2010). Creating the CIPRES science gateway for inference of large phylogenetic trees. In Proceedings of the Gateway, Computing Environments Workshop (GCE), pp. 1-8. New Orleans, LA.

Mogollon-Pasapera, E., Otvos, L., Giordano, A. and Cassone, M. (2009). Bartonella: emerging pathogen or emerging awareness? International Fournal of Infectious Diseases 13, 3-8.

O'Reilly, K. L., Bauer, R. W., Freeland, R. L., Foil, L. D., Hughes, K. J., Rohde, K. R., Roy, A.F., Stout, R. W. and Triche, P. C. (1999). Acute clinical disease in cats following infection with a pathogenic strain of Bartonella henselae (LSU16). Infection and immunity 67, 3066-3072. Pérez, C., Maggi, R., Diniz, P. and Breitschwerdt, E. B. (2011). Molecular and serological diagnosis of Bartonella infection in 61 dogs from the United States. Fournal of Veterinary Internal Medicine 25, 805-810. Pérez-Martínez, L., Venzal, J. M., González-Acuña, D., Portillo, A., Blanco, J. R. and Oteo, J. A. (2009). Bartonella rochalimae and other Bartonella spp. in fleas, Chile. Emerging Infectious Diseases 15, 1150-1152. Staggemeier, R., Venker, C. A., Klein, D. H., Petry, M., Spilki, F. R. and Cantarelli, V.V. (2010). Prevalence of Bartonella henselae and Bartonella clarridgeiae in cats in the south of Brazil: a molecular study. Memórias do Instituto Oswaldo Cruz 105, 873-878.

Statamakis, A., Hoover, P. and Rougemont, J. (2008). A rapid Bootstrap Algorithm for the RAxML Web Servers. Systematic Biology 57, 758-771.

Thrusfield, M. (2007). Veterinary Epidemiology, 3th Edn. Blackwell Science Ltd, UK.

Troncoso, I., Fischer, C., Arteaga, F., Espinoza, C., Azócar, T. and Abarca, K. (2016). Seroprevalencia de Bartonella henselae en personas con riesgo ocupacional. Revista Chilena de Infectologia 33, 355-357.

Zaror, L., Ernst, S., Navarrete, M., Ballesteros, A., Boroschek, D., Ferres, M. and Thibaut, J. (2002). Archivos de medicina Detección serológica de Bartonella henselae en gatos en la ciudad de Valdivia, Chile Serologic detection of Bartonella henselae in cats in the city of Valdivia. Archivos de Medicina Veterinaria 1, 103-110.

Zuñiga, M. L. (2007). Características Demográficas de la población canina y Recuento de la población felina, en la Ciudad de Valdivia, Chile. 